\title{
An Analysis of the Motion Pattern of a Bedside Exercise Rehabilitation Program Using a 3-axis Accelerometer
}

\author{
${ }^{1}$ Eun-Surk Yi, ${ }^{2}$ Ji-Youn Kim and ${ }^{3}$ Byung Mun Lee ${ }^{\dagger}$ \\ ${ }^{12}$ Dept. of Exercise Rehabilitation \& Welfare, Gachon University, 191, \\ Hambangmoe-ro, Incheon, South Korea \\ ${ }^{3}$ Dept. of Computer Engineering, Gachon University, 1342, Seongnam-daero, \\ Sujeong-gu, Seongnam-si, South Korea \\ ${ }^{1}$ yies@gachon.ac.kr, ${ }^{2}$ eve14jiyoun@naver.com, ${ }^{3}$ bmlee@gachon.ac.kr
}

\begin{abstract}
Systematic exercise is required for patients with degenerative joint conditions and the elderly in need of others' help. In this study, the authors developed 30 bed rehabilitation exercises for the elderly to conduct easily on a bed using their upper extremity muscles and fine motor muscles. However, the rehabilitation exercises are effective only if those who do them have the will to continue them. Many people are taking advantage of ICT, such as on their smartphones, for constant exercise management. In this paper, the authors suggested five different motions for bedside rehabilitation exercises and obtained experimental results from the motion patterns using a three-axis acceleration sensor of the smart phone. Each motion of the rehabilitation exercises has a specific pattern, which is composed of $x, y$, and $z$-coordinates from acceleration sensor of the smart phone. Further studies on the development of motion detectors/guides for assisting bedside rehabilitation exercises are needed.
\end{abstract}

Keywords: upper extremity muscles, fine motor muscles, motion pattern, 3-axis accelerator sensor

\section{Introduction}

The elderly population of Korea is $11.3 \%$ of the total population and $7 \%$ of city/province population. This rapid aging of the population leads to an increase in the incidence rate of senile dementia and chronic diseases, which in turn results in serious social problem [1]. One of the most typical senile diseases is stroke. Stroke is the secondbiggest cause of death in Korea and both the incidence rate and survival rate is on the increase [2].

More than $70 \%$ of stroke patients do not recover completely and experience hemiparalysis, which requires the help of other people to continue treatment [3]. Furthermore, intellectual disability and cognitive impairment including depression continue chronically [4]. For these patients, control of diseases and particularly the voluntary efforts of the patients are critical to postponing the incidence of complications. Unfortunately it is, however, known that $91 \%$ of the elderly with chronic diseases do not put rehabilitation exercise in practice on a regular basis [5].

According to the Korea Institute for Health and Social Affairs, 35\% of the elderly in Korea require the help of others to maintain their activities of daily living (ADL). Since ADL is closely associated with cognitive ability [6], the importance of movement is critical to preventing the secondary dementia for the elderly and patients with the problem with mobility.

${ }^{\dagger}$ Corresponding author 
Systematic joint exercise is critical to those in need of the help of others such as those with physical spasticity, degenerative joints and hemiparalysis. Particularly ADL focuses on the ability of upper limbs, so exercise of upper extremity muscles and fine motor muscles is essential.

Thus, in this study, the authors suggested bedside rehabilitation exercises for the patients and the elderly to use their upper extremity muscles and fine motor muscles. However it is important to continue to motivate the patients to maintain their will to continue exercise to ensure the effectiveness of the rehabilitation exercise on joint.

Recently U-health and mobile health are used as a way of motivation. U-health supports disease management and basic health management for elderly patients with chronic disease and disability (e.g. stroke) by means of remote rehabilitation and continued monitoring, showing its potential for preventive rehabilitation (e.g. musculoskeletal disorders, dementia, degenerative diseases, falls, etc.) and long-term rehabilitation and disease management (e.g. stroke, myocardial infarction, neurodegenerative diseases, brain cardiovascular diseases, etc.).

In this study, to apply the suggested bedside rehabilitation exercise to the mobile health, the motion patterns of bedside exercises were analyzed using 3-axis accelerometer built in smartphone, which is very important to develop wearable devices for bedside exercises in future.

In particular, the rehabilitation exercise using ICT has the advantage in accessibility and convenience [7-9], so its rapid feedback is expected to motivate the patients to conduct rehabilitation exercises regularly. Chapter 2 of this paper addresses related studies, such as studies on the basic concept of exercise rehabilitation and the measurement of the amount of exercise using sensor technology. Chapter 3 addresses the definition of the bedside exercise suggested in this paper. In Chapter 4, 5 key motions are selected from the suggested exercise motions and they are patterned and analyzed using a mobile phone motion sensor.

\section{Related Works}

\section{Basic Concepts of Exercise Rehabilitation}

Exercise is widely used for the purpose of leisure activities and health. Conventional exercise aims at the recovery of physical functions and the improvement of technical skills, but it shifts to the leisure sports in the 1990s. Its role is emphasized in view of recovery and rehabilitation [10]. In addition, exercise is closely associated with the rehabilitation therapy for disability and diseases. Exercise serves as a therapeutic approach to various diseases, which is named exercise therapy [11].

This concept of exercise is implicitly based on normal people. However in accordance with the National Health and Nutrition Examination Survey (2010), the incidence of adult diseases in Korea is the world's highest, and the increase of the suicide rate of teenagers and the elderly recorded the world's highest [12], implying that the exercise does not become a part of life and is not put into practice. In addition it is critical to have the disabled recover from their physical dysfunction and consequential difficulties of daily living. This is the purpose of exercise rehabilitation. As such, exercise rehabilitation refers to total health and welfare service programs aiming at physical, mental and social health through prevention and rehabilitation [13] and the subjects include all people who seek health. [10].

All printed material, including text, illustrations, and charts, must be kept within the parameters of the $815 / 16$-inch (53.75 picas) column length and $515 / 16$-inch (36 picas) column width. Please do not write or print outside of the column parameters. Margins are $15 / 16$ of an inch on the sides ( 8 picas), 7/8 of an inch on the top (5.5 picas), and $13 / 16$ of an inch on the bottom ( 7 picas). 
The rehabilitation program aims at gaining confidence and motivation through the movement of bodies and inducing voluntary participation. The purpose of exercise rehabilitation is to increase the psychological and social well-being and recover physical functions to live a life independently.

\section{Best Practices and Benefits}

To achieve the purpose of exercise rehabilitation program, many studies on the recovery of physical functions using various exercises are in progress in Korea and these programs put into practice. These programs are reported to have a positive effect on the recovery of physical functions [14], recovery of pulmonary functions [15], the cognitive ability of the elderly [16] and bone density and lipid concentration [17].

However, it is unfortunate that only the healthy elderly and those who have keen interest participated in such programs. The results of the studies on the exercise rehabilitation program with the weak and elderly over 75 who need of the help of others conducted in accordance with respective purpose of exercise rehabilitation program showed no significant variation in weight and BMI, yet indicated a positive effect in grip power, parallelism and cognitive and emotional elements pertaining to objective well-being.

In addition, the results of the studies on the exercise rehabilitation program with the runaway teenagers who used the shelter for teenagers showed a positive effect not only on mental health such as depression, anger and vitality but also on the secretion of growth hormone and serotonin [18]. However, to achieve the intended purpose of exercise rehabilitation program, the subjects need to continue to make efforts.

In recent years, many health management programs and apps for measuring the amount of exercise for smartphones have been developed and distributed. As the subjects are able to measure the amount of exercise and use the health information of their own using easy-to-carry smartphones, which motivates them to practice exercise rehabilitation.

\section{Measurement of the Amount of Exercise using a Sensor}

Most smartphones provide features to measure and manage the amount of exercise through walking exercise. Walking is widely used, ranging from weight management to the rehabilitation since it is a low impact aerobic exercise [19]. In recent years, with the development of smartphone with built-in acceleration sensor, apps to help walking exercise are increasingly developed and used [20,21].

This is possible because the pattern of the values measured by an accelerometer can measure and estimate various motions and behaviors of humans [22]. For example, as shown in Figure 1, the gait of a person creates a uniform pattern and the accelerometer measures this pattern in $\mathrm{x}, \mathrm{y}$ and $\mathrm{z}$ values according to the direction and the tilting angle of the person, and convert them into energy expression [22]. The use of these cyclic patterns can estimate the number of steps. 


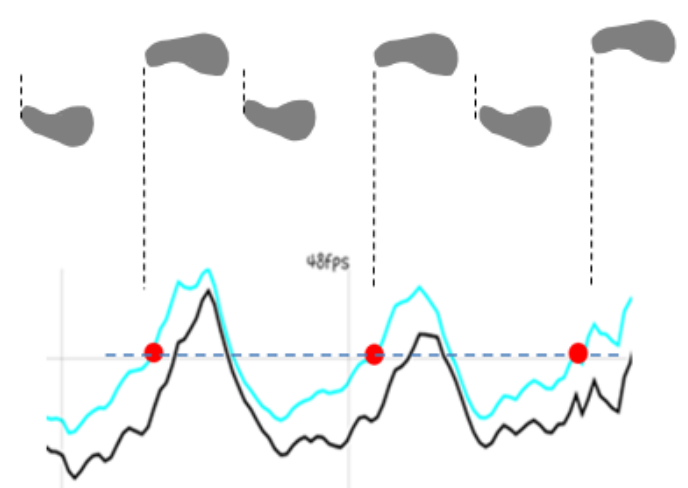

Figure 1. Variation of Sensor Pattern according to Gain Pattern

By multiplying the individual's stride by the estimated number of steps, the travel distance can be calculated and the amount of exercise can be obtained according to the travel distance. In addition, the gait pattern can be categorized according to the density of pattern period. In other words, the walking speed can be estimated as a slow pace, normal pace, quick pace, or a running pace. As such an accelerometer can cover the motion of humans into figures, so it can express the motion of rehabilitation exercise into figures too. This implies that the resilience type rehabilitation exercise suggested in this study can be digitized and patterned using an accelerometer.

\section{Suggestion of Bedside Rehabilitation Exercise}

The rehabilitation exercise suggested in this study is intended for the elderly or patient who are in need of help of others for daily ling. As the improvement of the functions of upper extremity is critical, this exercise is composed of the exercise for the joint and fine motor muscles of upper limbs and for motor coordination for walking. In addition, considering the limited space of hospital, this program is designed to be fit for a small space, i.e. bedside.

The program is largely composed of exercise for the entire body, fine motor muscles and coordination ability. Each exercise has 3 objectives and each object has 3 motions. Given the limited motion of elderly patients, maximum diversity and applicability were investigated and finally 30 motions were adopted. A double objective was set depending on the individual's ability and 12 to 15 motions were available to be chosen in the program. If there was a sign of adaptation and recovery, the choices and levels for motion increased as shown in Table 1 [23-24].

The motions used to develop this program include the motions related to the indexes pertaining to the evaluation of the ability of daily living (e.g. washing a face, eating, wearing or taking off clothes, relieving and bathing, etc.). In addition, this program is composed of the stages of exercise for upper extremities and fine motor muscles and coordination capability, emphasizing the rehabilitation to the activities of daily living.

Table 1. Self-bedside Exercise Program

\begin{tabular}{lcccc}
\hline \multicolumn{2}{c}{ Target of exercise } & \multicolumn{3}{c}{ Exercise } \\
\hline $\begin{array}{l}\text { Exercise of the } \\
\text { whole body }\end{array}$ & Relax & Stretching & $\begin{array}{c}\text { Shoulder } \\
\text { rotation }\end{array}$ & Relax \\
\hline $\begin{array}{l}\text { Exercise of the } \\
\text { whole body }\end{array}$ & Seat & Knee up & Arms up & Side flexion \\
\hline Exercise of the & Peripheral nerve & Tapping & Hand tapping & Wrist/ankle joint rotation \\
\hline
\end{tabular}




\begin{tabular}{|c|c|c|c|c|}
\hline whole body & & & & \\
\hline $\begin{array}{l}\text { Fine motor skills } \\
\text { (Bean-dumbbell) }\end{array}$ & Catch & Bouncing & $\begin{array}{c}\text { Putting on } \\
\text { back of } \\
\text { hand }\end{array}$ & Throw \\
\hline $\begin{array}{l}\text { Fine motor skills } \\
\text { (Bean-dumbbell) }\end{array}$ & Drop & Drop & $\begin{array}{l}\text { Turning back } \\
\text { of hand I }\end{array}$ & Drop - catch \\
\hline $\begin{array}{l}\text { Fine motor skills } \\
\text { (Bean-dumbbell) }\end{array}$ & Wring & Rubbing & $\begin{array}{c}\text { Turning back } \\
\text { of hand II }\end{array}$ & Twisting \\
\hline $\begin{array}{c}\text { Motor } \\
\text { coordination }\end{array}$ & Left \& right & Rock-paper & $\begin{array}{l}\text { Rock-scissors- } \\
\text { paper I }\end{array}$ & Rock-scissors-paper II \\
\hline \multirow{2}{*}{$\begin{array}{c}\text { Motor } \\
\text { coordination }\end{array}$} & Upper body & $\begin{array}{l}\text { Head-shoulder- } \\
\text { knee }\end{array}$ & $\begin{array}{l}\text { Drawing figure } \\
\text { of } 8\end{array}$ & Back drop \& catch \\
\hline & \multirow{2}{*}{$\begin{array}{l}\text { Upper \& lower } \\
\text { body }\end{array}$} & $\begin{array}{l}\text { Big shoulder } \\
\text { rotation }\end{array}$ & Ankle joint up & Hip walking \\
\hline $\begin{array}{c}\text { Motor } \\
\text { coordination } \\
\end{array}$ & & Seat knee up & $\begin{array}{c}\text { Seat rock- } \\
\text { scissors-paper }\end{array}$ & Seat stretching \\
\hline
\end{tabular}

The motions used to analyze the patterns are evenly adopted from the motion of whole body, fine motor muscles and coordination according to the category of program and the patterns were analyzed with focus on the motions of upper limbs.

The upper limb refers to body parts between the shoulder and wrist. The movement of upper limbs is associated with not only the humeri, radius, ulna, and related muscles, but also shoulders, cervical nerves and pectoral muscles, thereby giving the interoperability of movement. Particular the upper arms are connected to glenohumeral joints, facilitating distinct arm movement and largest working range of body part through the muscle of joining part, chest and neck. In conclusion, the working range of the shoulder joints connected to upper limbs facilitates the activities of daily living in most cases.

Elbows enable bending, stretching, pronation, supination, and strengthens the muscle of upper arms and improves the accuracy in the movement of arms.

Finally, the lower arms enable turning around the radius and ulna, being responsible for the bending and stretching of hands and fingers. In conclusion, to move the upper limbs which are closely associated with the activities of daily living, it is essential to strengthen the related muscles through bending, stretching and rotating of upper limbs, and multilateral kinesthetic stimuli is required to increase the flexibility of shoulder joint and wrist joint, which will in turn increase the working range.

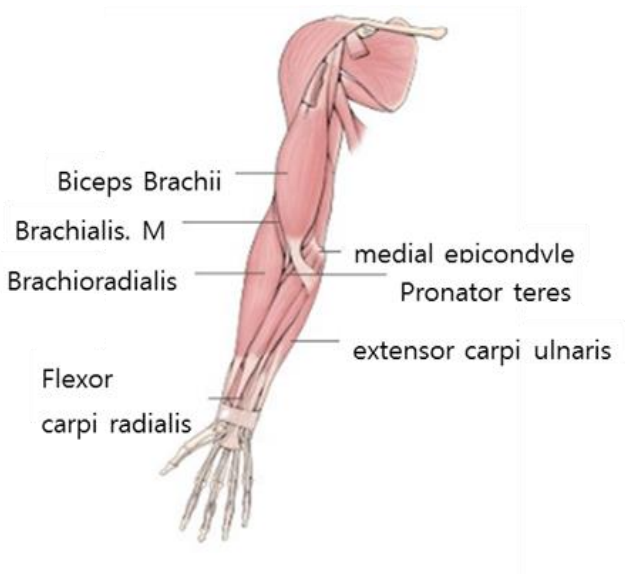

Figure 2. Anatomy of UpperLlimb 
Based on the above anatomical and functional theoretical background and items of ADL, the groups in need of the help of others were included in the recovery and rehabilitation program. To prove the possibility of analyzing exercise patterns using a 3-axis accelerometer, 5 motions were adopted as follows by considering the characteristics of the upper limbs: simple arm upper limbs exercise (a) Motion 1 upper arm joint exercise), upper limbs exercise including upper body (b) Motion 2flank exercise), turning exercise (c) Motion 3-twisting of arms), the movement of large joints (d) Motion 4- shoulder turn and coordination between the upper and lower body (e) Motion 5-head/shoulder/knees.

(a) Motion 1 - Upper arm joint exercise focuses on the strengthening of upper arm muscles and the movement of elbow. Important motion includes 'holding and lifting objects', 'eating' and 'washing a face' of ADL.

(b) Motion 2 - Flank exercise focuses on the strengthening of upper limbs joining and the strengthening and balancing of shoulders and upper body muscles with the aim to improve the working range of upper body and strengthen the muscles needed for seated posture.

(c) Motion 3 - Twisting of arms is the rotation of the whole upper limbs, improving the coordination between shoulder joint and elbow joint and the flexibility of them. This motion facilitates ADL (e.g. wearing and taking off clothes, using toilets, etc.)

(d) Motion 4 - Shoulder turn focuses on moving upper limbs (forward /backward/sideways) and increasing the movement of shoulder joints. Since this coordinated motion stimulates the abs and back muscles, increasing the abs and back muscle.

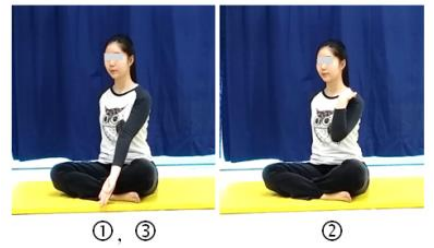

(a) Motion 1 - Upper arm joint exercise

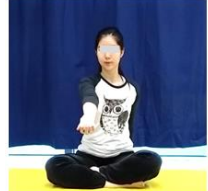

(1), (4)

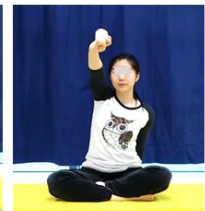

(2)

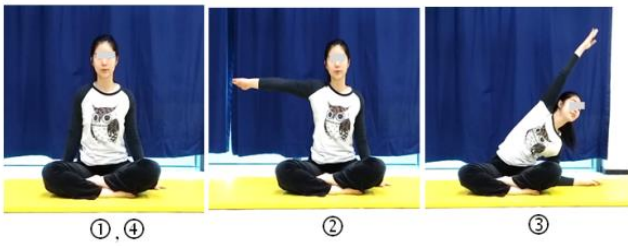

(b) Motion 2 - Flank exercise

(c) Motion 3 - Twisting of arms

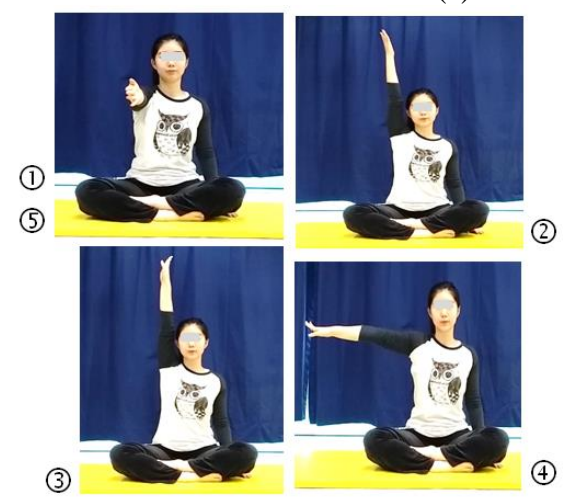

(d) Motion 4 - Large shoulder turn

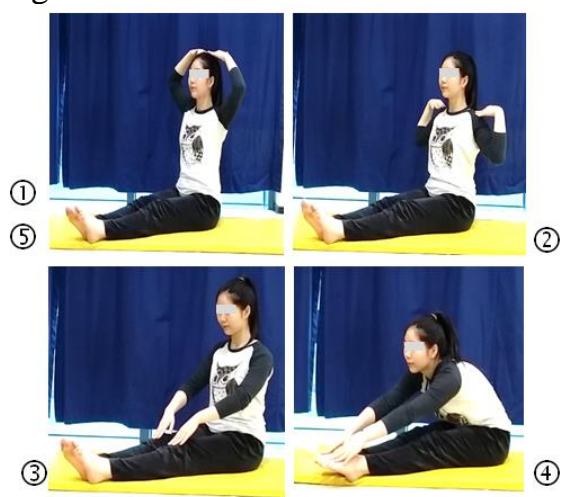

(e) Motion $5-$ Head/shoulder/knee

Figure 3. Five Key Motions of Rehabilitation Exercise 
(e) Motion 5 - In head/shoulder/knee exercise, the subjects touch each part of upper and lower body through the movement of upper limbs, which improves the flexibility, speed, agility and coordination of upper and lower limbs. This motion is very critical during the transition from bedside exercise to walking. The above 5 motions represent the purpose of this program and the functions of upper limbs and adequate for proving the possibility of continued monitoring through an analysis of motion patterns using a 3 -axis accelerometer.

\section{Experiments}

\section{Experimental Analysis of the Motion Patterns of Rehabilitation Exercise}

5 key motions of the rehabilitation suggested in this study were adopted. Figure 3 (a) shows a motion in which arms are raised (1) to the shoulder (2) and then lowered again(3).

Figure 3 (b) shows a motion in which right hand are raised horizontally(1), stretched to the left(2) and returned to its original position(4)). (c) is performed in the same order. (d) shows a motion in which a sand bag is held in right hand and raised horizontally in front (1), raised upward(2) in lotus posture, direction is changed(3) and then hand returns to its original position(4). (e) shows a motion in which both arms touch head(1), shoulders((2)), knees(3) and feet(4) in the same order.

To analyze the singularities of each motion through experiment, it is essential to measure the location of space for motion sensing. In this study, as shown in Figure 4, the variation of $\mathrm{x}, \mathrm{y}$ and $\mathrm{z}$ coordinate using 3-axis accelerometer built in smartphone and sensor app (Accelerometer Monitor). A total of 5 subjects repeated each motion 10 times and 50 tests by motion were conducted. Thus the authors could obtain a total 250 findings from 5 motions

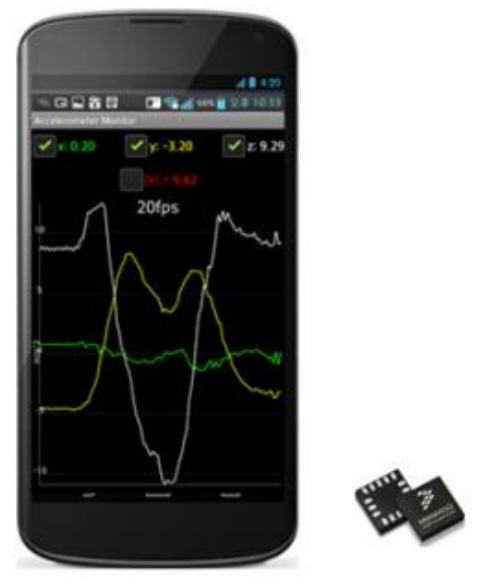

Figure 4. Smartphone's Accelerometer and Mobile Apps

\section{An Analysis of Experiment Results}

As shown in Figure 5(a), the variation of $x$-axis is not significant, whereas y-axis increases upward from 1 and decreases downward from 10. The $\mathrm{Z}$ value decreases downward from above 10 to -3 and then increases upward above 10. This is consistent with the pattern of motion - in which arms are raised and then lowered - measured by the accelerometer of the smartphone. There was a difference in values for each subject depending on the size of motion, yet 5 subjects showed identical pattern. 


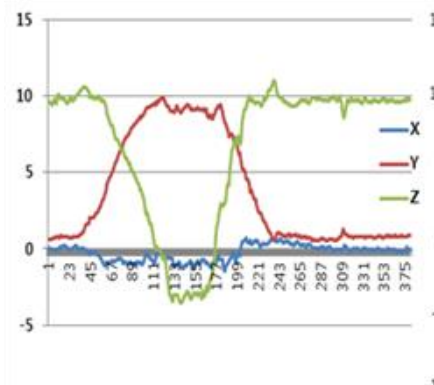

(a) Motion 1

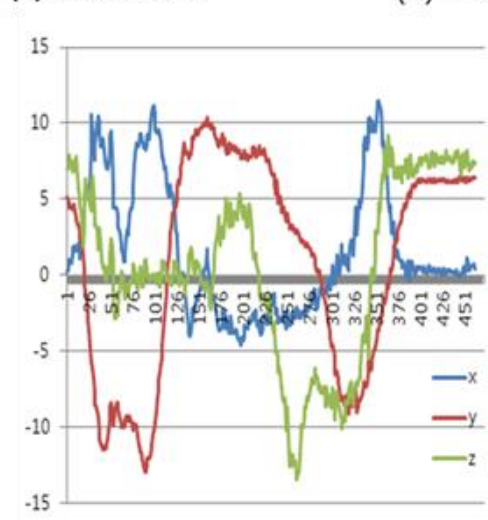

(d) Motion 4

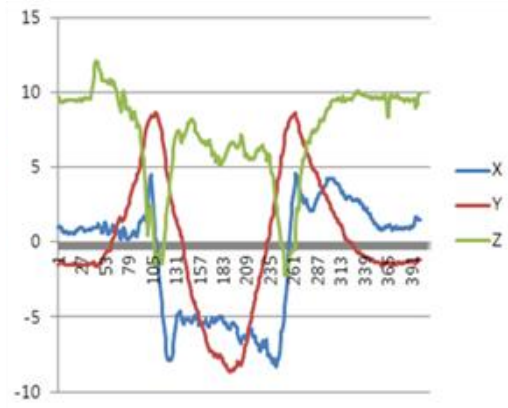

c) Motion 3

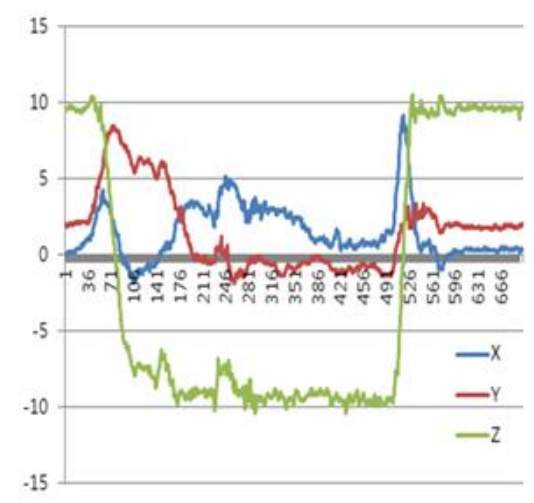

(e) Motion 5

\section{Figure 5. Five Key Motion Patters of Rehabilitation Exercise}

The $\mathrm{x}$-coordinate of motion (b) starts from 0 and increases upward to 8 and then decreases downward to -5 and then is restored to 0. As shown in Figure 3 (b), motion pattern and coordinate pattern show identical characteristics. The variation of $y$ coordinate shows a pattern opposite to that of $\mathrm{x}$-coordinate. In other words, y-coordinate starts from 5 and decreases to -8 , increases to 8 , decreases to -5 again and then is restored to 5. Looking at z-coordinate, its pattern is similar to that of y-coordinate, but it is crossed twice below 0 .

The $\mathrm{x}$-coordinate of motion (c) starts from 0 , increases to 4 and then sharply decreases to -81 . It continues to change within -5 , and increases to 5 and then is restored to 0 . Looking at the variation of $\mathrm{y}$-coordinate, it shows a pattern similar to that of the $\mathrm{x}$ coordinate. In other words, y-coordinate starts from 0 , increases to 8 , decreases to -8 . It increases to 8 again and then is restored to 0 . Looking at $\mathrm{z}$-coordinate, it changes from 10 to -1 and then decreases to -8 . It then increase to 8 , decrease to -1 and is restored to 0 . It show a pattern similar to $\mathrm{x}$-coordinate, but there is significant variation in the middle.

Analyzing the pattern graph of motion (d), it is irregular compared to Graph (a), (b), (c), so no singularity can be found. Looking at the pattern of $\mathrm{x}$-coordinate, it starts from 0 and increases upward twice and then sharply decreases to -4 and is maintained. It increases to 10 and then is restored to 0 . The y-coordinate shows a pattern opposite to the $\mathrm{x}$-coordinate. And $\mathrm{z}$-coordinate is very irregular compared to $\mathrm{x}$ and $\mathrm{y}$-coordinate, so not singularity can be found.

The pattern of motion (e) is very different from those of other motions. The $\mathrm{x}$ coordinate stays longer in the range of $0-5$ and the $y$-coordinate stays longer in the range of $0-5$. But the z-coordinate sharply decreases to -10 and stays in the range of -10 for a while and then sharply increases to 10 . 


\section{Conclusion}

This rapid aging of the population leads to an increase in the incidence of senile diseases. With the deterioration of cognitive ability and muscular functions, the elderly increasingly find it difficult to maintain their daily living without the help of others. Systematic joint exercise is critical to those in need of the help of others such as those with physical spasticity, degenerative joints and hemiparalysis. Particularly the activities of daily living focus on the ability of upper limbs, so exercise of upper extremity muscle and fine motor muscles is essential.

In this study, the authors divided the exercise into 3 categories: whole body exercise, fine motor muscle exercise and coordination ability exercise. Each had 3 objectives and each objective had 3 motions. As a result, a total of 30 motions for rehabilitation exercise were developed. Of them, the authors suggested 5 key motions by each objective.

However, to achieve the objective of exercise rehabilitation, the exercise needs to be conducted regularly. Most exercise requires continued efforts and strong will. In this study, to help conduct rehabilitation exercise, the authors perform an experiment to make patterns of exercise motions with $\mathrm{x}, \mathrm{y}$ and $\mathrm{z}$ coordinate measured during each motion using a smartphone 3-axis accelerometer. The findings of this study are expected to serve as the foundation to develop more advanced motion sensor for the exercise motions..

\section{Acknowledgements}

This work was supported by the National Research Foundation of Korea Grant funded by the Korean Government (NRF-2013S1A5B6044087).

\section{References}

[1] Statistics Korea, Editor, Annual Report on the cause of death statistics (2008).

[2] H. W. Park and E. J. Ryou, A Design Development of Hospitalized Patients' Pants for Bed-ridden Patients. J. of the Korean Society of Clothing and Textiles, vol. 32, no. 9, (2008) pp.1418-1426.

[3] F. D. Monahan and W. J Phipps, Phipps' Medical-Surgical Nursing Health \& Illness Perspectives. (2007), pp. 1428-1429.

[4] J. H. Kim, and T. R. Han, Editor, Rehabilitation Medicine. Seoul, Koonja published, (2014).

[5] K. H. Jung, The 2004 Survey on the Living Profile and Welfare Service Needs of Older Persons: Results and Policy Implications. Health and welfare policy forum, no. 101, (2005) pp. 49 - 65.

[6] H. S. Jang and H. J. Lee, Cognitive Function and Activity of Daily Living of Older Adults Using Longterm Care Service. Korean journal of health policy and administration, vol. 22, no. 4, (2012) pp. 522 537.

[7] R. J. Conejar and H. K. Kim, A Design of Mobile Convergence Architecture for U-healthcare International Journal of Software Engineering and Its Applications, vol. 9, no. 1, (2015) pp. 253-260.

[8] S. H. Lee and D. W. Lee, A Study on Review and Consideration of Medical Industry Convergence Based on U-healthcare. The Journal of digital policy \& management, vol. 11, no. 6, (2013) pp. 193 - 197.

[9] P. Meemasuk and C. Chantrapornchai, "On the Development of Nutrition Information Systems for Kidney Disease Patients", International Journal of Database Theory and Application, vol. 6, no. 3, (2013) pp. 33-38.

[10] M. R. Choi and E. S. Y, "Meta Analysis for Building up the concept of Sport Welfare in korea", Korean Journal of Exercise Rehabilitation, vol. 8, no. 3, (2012) pp. 3-15.

[11] I. H. Lee, T, S. Ko, Y. H. Kwon, K. Y. Kim and M. C. Kim, "Introduction to Physical Therapy", Tere Books (2008)

[12] The Korean Nutrition Society Dietary reference intakes for Koreans, Seoul, The Korean Nutrition Society, (2010).

[13] Korean Journal of Exercise Rehabilitation, Editor, Exercise Rehabilitation Management, Komoonsa. (2013)

[14] P. Y. Lee, "The Development and effect Verification of a Rehabilitation Gymnastics Program for PoorHealth Elderly People", Korean Journal of Exercise Rehabilitation, vol. 8, no. 1, (2013) pp. 27-36.

[15] H. W. Bae, "Effects of Rehabilitation exercise on Physical Functional Recovery of Hemiplegia Persons after Stroke", Bio-Medical Science, Korea University, (2007) 
[16] S. O. Choi, "The Effect of 24 weeks of Exercise Program on Cognitive Function and Health-Related Physical Fitness in Elderly Persons with Mild Dementia”, Korean journal of sports science, vol. 21, no. 6, (2012) pp.1087-1098.

[17] S. H. Kim and J. S. Kim, "Analysis of Correlations among Bone Mineral Density, Serum Lipid Levels, and Cognitive Function in the Elderly with Dementia", Journal of the Korean Society of Physical Medicine, vol. 7, no. 2, (2012) pp. 149 - 155.

[18] J. Y. Kim and K. S. Sim, "Effects of Rehabilitation Exercise gym program on physical fitness and subjective Well-being in Adult day care elderly", Korean Journal of Exercise Rehabilitation, vol. 8, no. 3, (2012) pp. 17-25.

[19] B. M. Lee, J. K. Kim, J. H. Kim and Y. H. Lee and W. K. Kang, “A Customized Exercise Service Model based on the Context-Awareness in u-Health Service", Journal of Korean institute of information technology, vol. 9, no. 2, (2011) pp. $141-152$.

[20] M. Alzantot and M. Youssef, "UPTIME: Ubiquitous Pedestrian Tracking using Mobile Phones", Proc. Of 2012 IEEE Wireless Communications and Networking Conference: Service, Applications, and Business, (2012) pp. 3204-3209.

[21] B. M. Lee, "Adaptation of Customized Measurement of Stride Length in Smart Device", The Journal of the Korea Contents Association, vol. 13, no. 4, (2012) pp. 35 - 43.

[22] K. Tumkur and S. Subbiah, "Modeling Human Walking for Step Detection and Stride Determination by 3-Axis Accelerometer Readings in Pedometer", Proc. of 4th Int'l Conference on Computational Intelligent, Modeling and Simulation, IEEE, (2013), pp. 199-204.

[23] Y. C. Lee, E. S. Yi, W. H. Choi, B. M. Lee, S. B. Cho and J. Y. Kim, "A study on the effect of self bedside exercise program on resilience and activities of daily living for patients with hemiplegia", Journal of Exercise Rehabilitation, vol. 11, no. 1, (2015) pp. 30-35

[24] J. Y. Kim, E. S. Yi and B. M. Lee, "Necessity of the development of Self exercise at bedside program", Science and Engineering Research Support society, Proceedings International Workshop Networking and Communication 2015, Mobile and Wireless 2015, (2015) pp. 6.

Authors

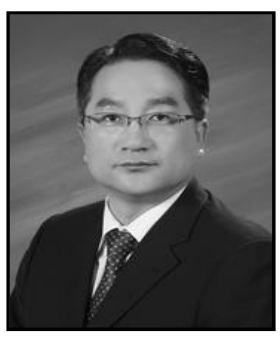

Eun-Surk Yi, He received a B.S., M.S., Ph.D., degree in 1993, 1998, 2003 from Korea National Sport, Seoul, Korea. He had worked for professor in the department of Gerokinesiology and Sports Industry, Daegu Haany University for 7 years Gyeongsangbuk-Do, Republic Korea. He is currently a professor in the department of Exercise Rehabilitation \& Welfare, Gachon University, Republic Korea. His research interests are sociology of sports and leisure, activity program protocol for elderly, leisure patterns, convergence Exercise Rehabilitation etc.

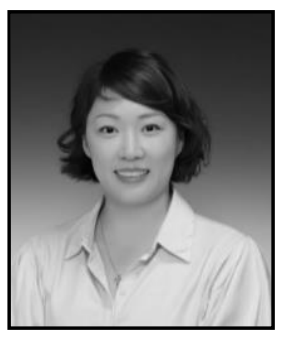

Ji-Youn Kim, She received a B.S. degree in 1998 from Kyusyu woman's College, Fukuoka, Japan and a M.S. degree from Fukuoka Education University and a Ph.D. degree from Sungkyunkwan University, in 2004 and 2008. He is currently a lecturer in the department of exercise Rehabilitation \& Welfare, Gachon University, South Korea. Her research interests are Exercise physiology, Sports nutrition, and health related exercise rehabilitation.

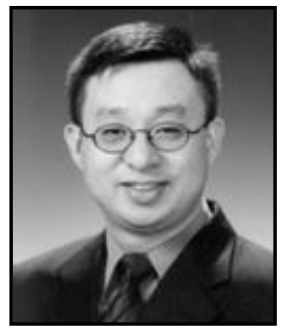

Byung Mun Lee, He received a B.S. degree in 1988 from Dongguk University, Seoul, Korea and a M.S. degree from Sogang University and a Ph.D. degree from Incheon National University, in 1990 and 2007. He had worked for LG Electronics for 7 years and was a visiting scholar professor at California State University Sacramento, USA. He is currently a professor in the department of Computer Engineering, Gachon University, South Korea. His research interests are pervasive healthcare, its network protocol, IoT for healthcare, wireless sensor networks, operating system, etc. 